\title{
Special Issue: Contemporary development of neural computation and applications
}

\author{
Ivan Jordanov $\cdot$ Bruno Apolloni $\cdot$ Nikola Kasabov
}

Published online: 21 March 2012

(C) Springer-Verlag London Limited 2012

The aim of this Special Issue is to solicit theoretical and application-oriented research in the field of neural computing and to present examples of experimental and real-world investigations that demonstrate the advances, successes, and state-of-the-art of the developments in the neural computing area. We believe it provides a good opportunity for reflection on current developments in neural network research and its applications and gives an impression of where the research in the included topics is headed.

From the submitted 57 manuscripts, the review process selected 10 papers, which in our view reflected in the best way the aim and objectives of the Issue, representing a mixture of cross-cutting investigations and in-depth articles that give a snap shot of current developments in the field. The presented authors cover a variety of topics related to neural computing and implementations of nature-inspired computational models that include the following:

- an adaptive neural network-based algorithm used to significantly improve the visual quality of JPEGcompressed images and videos;

- time series forecasting by evolving artificial neural networks with genetic algorithms, differential evolution, and estimation of distribution algorithm;

I. Jordanov $(\bowtie)$

University of Portsmouth, Portsmouth, UK

e-mail: ivan.jordanov@ port.ac.uk

\section{B. Apolloni}

University of Milan, Milan, Italy

e-mail: apolloni@dsi.unimi.it

N. Kasabov

Auckland University of Technology, Auckland, New Zealand

e-mail: nkasabov@aut.ac.nz
- a support vector machine-based iterative Markovswitching multifractal model for financial short-term volatility forecasting;

- a new classification technique for electroencephalogram (EEG) signals and a real-time EEG in a real-time brain-computer interface application for a mobile robot control using fuzzy functions support vector classifiers, particle swarm optimization (PSO), and radial basis function networks;

- theoretical investigation of conditions for global exponential stability of continuous-time neural networks with delays, employing suitable Lyapunov functional;

- a study of how to build ensemble classifiers from fuzzy ART and SOM networks for which parameters tuning a hybrid metaheuristic based on PSO and simulated annealing (SA) are used;

- investigation of learning gene regulatory networks using swarm intelligence bees optimization algorithm to train networks with predefined attractors, which shows better results when compared with SA algorithm;

- a novel weight pruning method for MLP classifiers based on the observation that non-relevant synaptic weights tend to generate smaller correlations between error signals associated with the neurons of a given layer and the propagated error signals back to the previous layer;

- an application of neural networks for fetal monitoring in labor, which is very important for preventing birth asphyxia that can result in death or permanent brain damage of newborn babies. This work also uses principal component analysis to reduce the feature space dimensionality of the training data;

- investigation of negative correlation learning method that employs special error function for the simultaneous training of base neural network experts, which 
demonstrates improved performance when compared with competitive learning approach (winner-takes-all) and average combining techniques.

As we mentioned above, the Special Issue aimed to communicate ideas among a diverse group of scientists and researchers who share common interest in nature- and biology-inspired methodologies in general and in neural networks in particular. We leave to the reader to judge whether the aim is met, but hope they will agree with us that the published papers reflect convincingly the Issue's objectives with its variety of presented topics, investigated in both theoretical and application levels. 